\title{
LA TUTELA DELLA VITA E DELL'INTEGRITÀ PERSONALE NEL SISTEMA PENALE ITALIANO
}

\section{Pasquale Gianniti}

Consigliere della Corte di Cassazione (Italia)

pasquale.gianniti@gmail.com

\section{Rilevanza costituzionale del diritto alla vita ed alla incolumità individuale}

La Costituzione italiana, a carattere personalistico-solidaristico, pone, al proprio centro, la persona umana, nella sua unitarietà fisico-psichica e nella sua dimensione individuale-sociale (artt. 2 e 3). Essa riconosce i diritti inviolabili della persona umana ed è tesa a favorirne il pieno sviluppo mediante l'imposizione: alla Repubblica, della rimozione degli ostacoli, limitativi, di ordine economico e sociale; e, ai singoli, dell'adempimento dei doveri inderogabili di solidarietà politica, economica e sociale.

In un ordinamento, così caratterizzato, il diritto alla vita costituisce il bene-fine primario. ${ }^{1}$

Per un apparente paradosso, il diritto alla vita non è espressamente riconosciuto dalla Carta costituzionale italiana, contrariamente a quanto avviene in altre Costituzioni (ad es., tedesca, spagnola e portoghese), nella Convenzione europea dei diritti dell'uomo e nella Carta dei diritti fondamentali dell'Unione Europea.

Tuttavia, nel sistema costituzionale italiano il diritto alla vita si desume in-

1 Si ricorderà che il primo grande scrittore politico che formulò la tesi del contratto sociale, Tommaso Hobbes, riteneva che il diritto alla vita è l'unico diritto cui i contraenti, entrando in società, non rinunciano. Secondo Hobbes, i cittadini rinunciano a difendersi da soli, prevedono la soppressione delle bande armate e degli eserciti locali, attribuiscono allo Stato il monopolio dell'uso della forza, affinché quest'ultimo svolga un ruolo pacificatore soprattutto a difesa della vita e dell'integrità personale di ciascuno di fronte ad aggressioni, esterne ed interne. Dunque, il desiderio di garantire la protezione della vita è la ragione d'essere del patto politico. E lo Stato nasce per l'appunto per garantire beni come la vita, la libertà, l'uguaglianza, la proprietà e la salute

E si ricorderà altresì che Sant'Agostino affermava che, se nella comunità pubblica prevale la corruzione, sarebbe impossibile distinguerla da una banda di ladri. 
equivocabilmente: dagli artt. 13 comma 4 e 32 Cost., che tutelano la vita e l'integrità fisica e psichica della persona; dall'art. 27 comma 4, che pone il divieto di uccidere anche ai pubblici poteri ed anche nei confronti degli autori dei delitti più gravi; dalle diverse norme della legislazione, penale e civile, che tutelano il diritto alla vita come interesse individuale alla propria sopravvivenza (artt. 575 c.p., nonché artt. 5, 443, 2043, 2087 c.c.).

Il fatto è che il diritto alla vita rientra nei beni-presupposto della Costituzione: senza il riconoscimento e la tutela del diritto alla vita sarebbero privi di significato il riconoscimento e la tutela di tutti gli altri diritti individuali e sociali della persona umana, come pure l'imposizione della rimozione dei suddetti ostacoli e dell'adempimento dei suddetti doveri.

Il diritto alla vita, anzi, tramite il disposto dell'art. 2 Cost., è il diritto inviolabile per eccellenza.

Bene fine primario, anche se di rango inferiore al bene della vita, è poi il diritto alla incolumità individuale, cioè il diritto a godere del proprio stato di salute, della propria funzionalità psico-fisica e della propria funzionalità estetica, a prescindere dal grado in cui detti beni si manifestino in concreto nella vita di ciascuna persona umana.

In tale accezione ampia l'incolumità individuale è costituzionalmente tutelata: dall'art. 32, che eleva la salute a diritto fondamentale dell'individuo; dall'art. 13, che tutela la disponibilità dell'essere corporeo da ogni intervento coercitivo statuale; nonché dagli artt. 3 comma 1, 27 comma 2, 32 comma 2 e 42 comma 2 , che consentono di enucleare la dignità umana come valore costituzionale (elevato, anch'esso, tramite l'art. 2, alla categoria di diritto inviolabile).

Oggetto delle presenti note è la disamina comparativa delle tre fattispecie criminose, che sono di per sè dirette alla tutela del bene dell'integrità personale e della vita: il reato di percosse, il reato di lesioni ed il reato di omicidio.

Tra le suddette tre fattispecie criminose è ravvisabile una inquietante progressione criminosa. Il soggetto passivo: nel primo, viene percosso; nel secondo, viene leso; nel terzo, viene privato della vita.

\section{Percosse e lesioni personali}

\subsection{II delitto di percosse}

Il delitto di percosse, previsto dall'art. 581 c.p., consiste nel fatto di chiunque "percuote" taluno, "se dal fatto non deriva una malattia nel corpo o nella mente". La condotta del percuotere comprende non soltanto il battere ed il colpire 
il corpo altrui, ma anche l'assoggettamento del corpo altrui ad una qualsiasi azione violenta, idonea a cagionare una sensazione dolorosa: l'atto di percuotere può realizzarsi attraverso qualsiasi forma (ad es., schiaffo, sculacciata, pugno, calcio, bastonata, urto, spinta, afferramento, scuotimento violenti; getto di cosa, trascinamento per i capelli; puntura con un ago o siringa, ecc.) e con mezzi di offesa naturali (mani, testa, piedi, ecc.) o artificiali (sassi, bastoni, libri, ecc.).

\subsection{Percosse e lesioni volontarie}

Il delitto di percosse si distingue dal delitto di lesione volontarie, che è previsto dagli artt. 582 e 590 c.p. e che consiste nel fatto di chiunque cagioni ad altri una lesione dalla quale derivi "una malattia nel corpo o nella mente".

I due delitti hanno in comune il fatto di essere delitti contro l'incolumità individuale (e, quindi, di tutelare entrambi l'integrità della persona contro qualsiasi aggressione che si traduca in una violenza sul corpo).

Tuttavia, si differenziano sotto plurimi profili. In primo luogo, sul piano oggettivo, in quanto il delitto di cui all'art. 581 c.p. è configurabile allorquando la violenza produce al soggetto passivo soltanto una sensazione fisica di dolore, ${ }^{2}$ senza postumi di alcun genere; mentre il delitto di cui all'art. 582 c.p., che pure può essere commesso con qualsiasi mezzo, sussiste quando il soggetto attivo cagioni al soggetto passivo una lesione, dalla quale derivi una malattia nel corpo o nella mente. ${ }^{3}$ Inoltre, sempre sul piano oggettivo, soltanto il delitto di percosse è reato necessariamente commissivo, in quanto una condotta omissiva non sarebbe comunque idonea a concretare un atto violento contro l'altrui persona. ${ }^{4}$ Infine, soltanto il delitto di percosse può essere reato di mera condotta, in quanto, per la sua sussistenza, non è necessaria la verificazione dell'evento della sensazione fisica dolorosa (anche se normalmente questa ricorre), ma è sufficiente che la condotta sia obiettivamente idonea (per il suo con-

2 La sensazione di dolore può anche non ricorrere (come nel caso di chi è stato percosso mentre si trovava in uno stato di anestesia o di insensibilità della parte del corpo attinta dalle percosse: cfr. Cass. pen., Sez. 1, sent. n. 7388 del 11/06/1985). Può essere utile ricordare che in passato, l'atto di percuotere, se idoneo (non a provocare dolore, ma soltanto) a umiliare o a mortificare la vittima, era astrattamente riconducibile all'oggi abrogato reato di ingiuria (si pensi al buffetto dato in pubblico soltanto per scherno).

3 Cass. pen., Sez. 5, sent. n. 714 del 15/10/1998 (in fattispecie, nella quale la Corte ha ritenuto sussistenti le lesioni per difficoltà respiratorie, durate alcuni minuti, a seguito di stretta al collo e scuotimento della vittima).

4 E' configurabile il tentativo qualora si dimostri che gli atti, in concreto posti in essere dal soggetto agente, erano diretti a realizzare una percossa dalla quale non sarebbe derivata una malattia. 
tenuto apprezzabile di violenza), secondo l'id quod plerumque accidit a produrre una siffatta sensazione.

I due reati si distinguono anche sotto il profilo soggettivo: il delitto di percosse è punibile soltanto a titolo di dolo, ${ }^{5}$ mentre il delitto di lesioni è punibile anche a titolo di colpa.

Altre differenze concernono il profilo processuale: il delitto di percosse è punibile soltanto a querela della persona offesa; l'arresto, il fermo e le misure cautelari non sono consentiti; la competenza, a seconda dei casi, ${ }^{6}$ è del giudice di pace o del tribunale monocratico.

\subsection{Rapporti con altri reati}

Quanto infine ai rapporti con altri reati commessi con violenza, l'art. 581 comma 2 stabilisce che la norma sulle percosse "non si applica quando la legge considera la violenza come elemento costitutivo o come circostanza aggravante di altro reato": si tratta cioè dei reati che prevedono la violenza come elemento costitutivo necessario (come la violenza privata, la rapina e l'estorsione) ovvero meramente eventuale (come l'abuso dei mezzi di correzione o di disciplina, i maltrattamenti contro familiari o conviventi e l'omicidio).

Ciò si giustifica con il fatto che in tali casi ci si trova di fronte ad ipotesi di reato complesso, che già contiene le percosse. Lo stesso può, altresì, accadere quando ci si trovi di fronte ad un altro delitto, che non può essere commesso se non attraverso delle percosse, le quali, pertanto, risultano assorbite.

5 Il soggetto agente deve cioè agire con la coscienza e la volontà di tenere una condotta violenta, astrattamente idonea a provocare una sensazione dolorosa al soggetto passivo. Nessuno, quindi, può essere chiamato a rispondere del reato di percosse per il fatto di aver accidentalmente colpito altri: si pensi a chi ha alzato di scatto il braccio per fornire un'indicazione e, così facendo, ha colpito in pieno volto un passante; oppure a colui che, facendo jogging per strada, urta un pedone, facendolo rovinare a terra. In tali ipotesi non è ravvisabile la volontarietà della condotta, ragion per cui il soggetto agente non potrà essere chiamato a rispondere del reato di percosse.

6 A partire dal 2 gennaio 2002, data di entrata in vigore del d.lgs. n. 274/2000, il delitto di percosse è passato alla competenza del Giudice di Pace, il quale applica la pena della sola multa da 258 a 2.582 euro (ex art. 52 comma 2 lett. a), d.lgs. n. 274/2000), salvo che non ricorrano le circostanze aggravanti indicate dall'art. 4 del decreto, poiché in tal caso il reato rientra nella competenza penale del tribunale monocratico e trova applicazione la cornice edittale (reclusione fino a 6 mesi o multa fino a 309 euro) prevista dall'art. 581 c.p. 


\section{Lesioni personali ed omicidio}

\subsection{Il delitto di lesioni personali ed il concetto di malattia}

Il delitto di lesioni personali, si diceva, consiste nel fatto di chiunque cagioni in altri una lesione dalla quale derivi "una malattia nel corpo o nella mente".

Ma che cosa è la malattia, richiesta per la sussistenza del reato di lesioni?

Secondo la Relazione ministeriale dei lavori preparatori del c.p., è malattia qualsiasi alterazione, anatomica o funzionale, dell'organismo, ancorchè localizzata e non influente sulle condizioni organiche generali.

Tale nozione tecnico giuridica del concetto di malattia non è stata condivisa dalla prevalente dottrina, in quanto il codice penale, nel distinguere le fattispecie di lesioni soprattutto in base alla durata della malattia, dimostra di accogliere la nozione di malattia non come "stato", ma come "processo morboso", cioè come una successione di fenomeni, che ha un inizio e, prima o poi, un esito (che può essere la guarigione perfetta, l'adattamento a nuove condizioni di vita oppure la morte). Pertanto, ai fini della sussistenza del reato di lesioni, non è sufficiente una qualsiasi alterazione anatomica o funzionale, se irrilevante (come ad es. per le ecchimosi o contusioni, per le abrasioni, per svenimenti di brevissima durata).

D'altra parte, secondo la giurisprudenza di legittimità, ${ }^{7}$ ai fini della configurabilità del delitto di lesioni personali, la nozione di malattia non comprende tutte le alterazioni di natura anatomica (che possono anche mancare), bensì solo quelle da cui deriva una limitazione funzionale o un significativo processo patologico (o un suo aggravamento) ovvero una compromissione delle funzioni dell'organismo, anche non definitiva, ma comunque significativa. Dunque, il concetto clinico di malattia richiede il concorso di un duplice requisito: da un lato, una riduzione apprezzabile di funzionalità, a cui può anche non corrispondere una lesione anatomica, e, dall'altro, un fatto morboso in evoluzione (a breve o lunga scadenza, verso un esito che potrà essere la guarigione perfetta, l'adattamento a nuove condizioni di vita oppure la morte). Ne consegue che non costituiscono malattia (e, quindi, non possono integrare il reato di lesioni personali), le alterazioni anatomiche, a cui non si accompagni una riduzione apprezzabile della funzionalità. ${ }^{8}$

7 Cass. pen., Sez. 5, sent. n. 33492 del 14/05/2019 (fattispecie relativa ad aggressione consistita in una "tirata di capelli", nella quale la Corte ha annullato con rinvio la decisione di merito che si era limitata a dar conto del referto medico che riportava, quale conseguenza a carico della vittima, "dolore in regione occipitale guaribile in giorni due").

In questi termini, ad es., la già citata sent. n. 714/1998. 
Si ritiene comunemente che la durata della malattia persista fino a quando permane il processo patologico di difesa o restaurazione dell'organismo, cioè fino alla data della guarigione clinica, che va determinata secondo rigorosi accertamenti medico-legali. Conseguentemente, nella durata della malattia, vengono tradizionalmente compresi anche il periodo di convalescenza o di riposo, dipendente dalla malattia stessa, e le varie manifestazioni terminali. ${ }^{9}$

I postumi sono le eventuali conseguenze della malattia, clinicamente guarita. I postumi temporanei rientrano nella malattia, ogni qualvolta determinino rilevanti disturbi funzionali (ad es., l'indebolimento temporaneo di un organo). Al contrario, i postumi permanenti (ad es., la perdita di un arto) non rientrano nella malattia, ma sono previsti come lesioni gravi o gravissime.

\subsection{Lesioni personali dolose e lesioni personali colpose}

Il delitto di lesioni personali è previsto: dall'art. 582, nella forma dolosa; e dall'art. 590 c.p., nella forma colposa.

Giova premettere che il vigente codice penale italiano prevede quattro forme di lesioni personali dolose, mentre prevede tre forme di lesioni personali colpose. Invero, le lesioni dolose possono essere lievissime, ${ }^{10}$ lievi, ${ }^{11}$ gravi $^{12}$ e gravissime $;{ }^{13}$

9 Ad es. manifestazione terminale è, nel caso di frattura, il consolidamento del calleo osseo e, nel caso di ferita, il venir meno della crosta ematica.

10 La lesione dolosa lievissima, prevista dall'art. 582 comma 2 (e procedibile a querela di parte), consiste nell'aver cagionato una malattia di durata non superiore a 20 giorni, in assenza di qualunque degli eventi previsti dall'art. 583, nonché di qualsiasi circostanza aggravante, prevista negli artt. 576 e 577 (ad eccezione di quelle indicate nel n. 1 nell'ultima parte dell'art. 577: delitti commessi contro l'ascendente o il discendente, il coniuge, il fratello o la sorella, il padre, la madre o il figlio adottivi o contro un affine in linea retta).

11 La lesione dolosa lieve, desumibile dal combinato disposto degli artt. 582 comma 1 e 583 comma 1 numero 1, consiste nell'aver cagionato una malattia di durata superiore ai 20 giorni ma non superiore a 40 giorni.

12 La lesione dolosa grave, prevista dall'art. 583 comma 1 e comma 2, consiste nell'aver cagionato una malattia (o una incapacità di attendere alle ordinarie occupazioni) che abbia durata superiore ai 40 giorni ovvero che metta in pericolo la vita della persona ovvero determini l'indebolimento permanente di un senso o di un organo.

13 La lesione dolosa gravissima, prevista dall'art. 583 comma 2, consiste nell'aver cagionato: una malattia certamente o probabilmente insanabile; la perdita di un senso; la perdita di un arto (o la mutilazione che renda l'arto inservibile); la perdita dell'uso di un organo o della capacità di procreare; una permanente e grave difficoltà della favella; la deformazione ovvero lo sfregio permanente del viso. 
mentre le lesioni colpose possono essere lievi, ${ }^{14}$ gravi ${ }^{15}$ e gravissime. ${ }^{16}$

Le suddette 7 figure di reato si distinguono tra loro:

a) in base alla durata della malattia (si considerino i disposti degli artt. 582 comma 2 e 583 comma 1 numero 1 );

b) in base all'alterazione anatomico-funzionale (si consideri il disposto dell'art. 583 comma 2 numeri 2, 3 e 4). ${ }^{17}$

Orbene, le lesioni personali dolose (art. 582) e le lesioni personali colpose (art. 590) hanno in comune, sotto il profilo oggettivo, il cagionare in altri una lesione, dalla quale derivi una malattia nel corpo o nella mente. Non è richiesta la violenza fisica, in quanto il delitto può essere realizzato anche con un'azione non violenta (quale l'esposizione del neonato al freddo ovvero la somministrazione di cibi insalubri) o financo con mezzi psichici (quale una grave minaccia, che abbia l'efficacia di perturbare la mente del soggetto passivo).

Tuttavia, si distinguono sotto diversi profili. In primo luogo, l'elemento soggettivo richiesto nelle lesioni personali dolose è per l'appunto il dolo (cioè la consapevolezza da parte del soggetto agente che la propria condotta è astrattamente idonea a provocare danni fisici alla vittima), anche in forma eventuale; mentre l'elemento soggettivo nelle lesioni personali colpose è rappresentato dalla colpa dell'agente, anche in forma cosciente, ${ }^{18}$ nella verificazione dell'evento.

14 La lesione colposa lieve consiste nell'aver cagionato una malattia di durata non superiore a 40 giorni (dunque, la lesione colposa lieve corrisponde, quanto a durata, alle lesioni dolose lievissime ed alle lesioni colpose lievi).

15 La lesione colposa grave consiste in uno degli eventi previsti dall'art. 583 comma 1 (sopra indicati).

16 La lesione colposa gravissima è quella che consiste in uno degli eventi previsti dall'art. 583 comma 2 (sopra indicati).

17 Le pratiche di mutilazione degli organi genitali femminili (cioè quelle pratiche che comportano la rimozione, parziale o totale, dei genitali esterni femminili, compiute per motivazioni culturali o per altre motivazioni non terapeutiche) costituiscono condotte criminose, che in passato erano punibili come lesioni dolose gravi o gravissime e che oggi integrano invece autonomi reati, previsti dagli artt. 583 bis comma 1 e comma 2. Detti reati - che sono stati introdotti dalla legge 9 gennaio 2006, n. 1 - consistono nel cagionare una mutilazione degli organi genitali femminili, in assenza di esigenze terapeutiche (comma 1); e nel provocare, sempre in assenza delle suddette esigenze, al fine di menomare le funzioni sessuali, lesioni agli organi genitali femminili diverse da quelle indicate nel primo comma, da cui derivi una malattia nel corpo o nella mente (comma 2). Il legislatore italiano ha voluto rendere oggetto di una autonoma previsione, più severamente sanzionata, le suddette pratiche al fine di sottolinearne il disvalore e di precludere al giudice la possibilità di operare il bilanciamento con le circostanze attenuanti.

Per analoghe considerazioni con legge 23 marzo 2016 n. 41 è stato introdotto l'art. 590 bis, che prevede le lesioni stradale come autonomo reato colposo.

18 Il soggetto agente, sia nel caso del dolo eventuale che nel caso della colpa cosciente, si rappresenta la possibilità della realizzazione del fatto. Tuttavia, egli, nel caso di dolo eventuale, accetta il rischio della realizzazione del fatto (che dunque vuole), mentre, nel caso della colpa cosciente, agisce con la sicura fiducia che il fatto (che dunque non vuole) non si realizzerà. 
Inoltre, la lesione dolosa, in tutte le sue forme, conosce le stesse circostanze aggravanti speciali previste per l'omicidio preterintenzionale; mentre la lesione colposa, se grave o gravissima, conosce le stesse circostanze aggravanti speciali previste per l'omicidio colposo.

Infine, le circostanze aggravanti, previste dagli artt. 583 e 585, sono circostanze aggravanti speciali del reato di lesione dolosa (e non figure autonome di reato), con conseguente applicabilità del giudizio di bilanciamento previsto dall'art. 69. Al contrario, le tre figure di lesioni personali colpose, previste dall'art. 590, costituiscono fattispecie autonome di reato, che si differenziano tra loro in base alla gravità delle lesioni prodotte ed alla diversa intensità dell'elemento soggettivo.

\subsection{Lesioni personali ed omicidio}

Il reato di lesioni personali, al pari dell'omicidio, è reato a forma libera, in quanto è integrato da qualsiasi comportamento (attivo od omissivo, anche non violento) idoneo a cagionare l'evento della malattia, rispetto al quale oggetto materiale è sempre e soltanto la persona umana. Inoltre, entrambi ammettono il tentativo. ${ }^{19}$

Il reato di lesioni personali ed il reato di omicidio si distinguono perché offendono beni giuridici diversi: l'omicidio offende il bene della vita, mentre le lesioni personali offendono il bene della incolumità individuale. Inoltre, diverso è l'evento, che, nel caso dell'omicidio, è la morte, mentre, nel caso delle lesioni, è la malattia nel corpo o nella mente.

\subsection{Lesioni personali dolose e tentato omicidio}

Delicati sono infine i confini tra le lesioni personali dolose ed il tentativo di omicidio. $^{20}$

19 La giurisprudenza di legittimità è ormai da anni consolidata nel ritenere che il dolo eventuale non è compatibile con il delitto tentato (Cass. pen., Sez. 1, sent. n. 25114 del 31/03/2010), ma deve qualificarsi come dolo diretto, e non meramente eventuale, quella particolare manifestazione di volontà dolosa definita dolo alternativo, che sussiste quando il soggetto attivo prevede e vuole, con scelta sostanzialmente equipollente, l'uno o l'altro degli eventi (nella specie, morte o grave ferimento della vittima) causalmente ricollegabili alla sua condotta cosciente e volontaria, con la conseguenza che esso ha natura di dolo diretto ed è compatibile con il tentativo (Cass. pen., Sez. 1, sent. n. 27620 del 24/05/2007).

20 Trattasi di questione di grande rilievo pratico, in quanto, nel caso di lesioni aggravate (art. 583 comma $1^{\circ}$ ), la pena astrattamente irrogabile va da tre a sette anni di reclusione; mentre, nel caso di 
Per giurisprudenza costante della Corte di legittimità, ${ }^{21}$ ai fini della diversa definizione del fatto materiale nel reato di lesione personale dolose e in quello di tentato omicidio -così come avviene in genere per tutti i casi di reato progressivo- deve aversi riguardo:

a) al diverso atteggiamento psicologico dell'agente;

b) alla differente potenzialità dell'azione lesiva.

Sotto detto ultimo profilo, nel reato di lesione personale dolosa l'azione esaurisce la sua carica offensiva nell'evento prodotto, mentre nel tentato omicidio vi si aggiunge un quid pluris che, andando al di là dell'evento realizzato, tende ed è idoneo a causarne uno più grave in danno dello stesso bene giuridico o di un bene giuridico superiore, riguardanti il medesimo soggetto passivo, non riuscendo tuttavia a cagionarlo per ragioni estranee alla volontà dell'agente.

Quanto invece all'atteggiamento psicologico dell'agente, l'operazione logica, diretta a trarre l'atteggiamento psichico del ricorrente dalle modalità dell'azione e dal mezzo impiegato, è la metodica comunemente utilizzata per verificare l'intenzionalità omicidiaria nel tentativo, ricerca da condurre secondo il criterio della prognosi postuma ex ante.

In tali termini è il consolidato orientamento della Corte di legittimità, ${ }^{22}$ secondo la quale, in tema di omicidio tentato, la prova del dolo, in assenza di esplicite ammissioni da parte dell'imputato, ha natura indiretta, sicchè deve essere desunta da elementi esterni e, in particolare, da quei dati della condotta che, per la loro inequivoca potenzialità offensiva, siano i più idonei ad esprimere il fine perseguito dall'agente (quali i mezzi usati; la direzione e 1 'intensità dei colpi; la distanza del bersaglio; la parte del corpo attinta; le situazioni di tempo e di luogo che favoriscano l'azione cruenta).

\section{L'omicidio nelle sue diverse forme 4.1.Tre figure di omicidio comune}

Il codice penale prevede tre figure di omicidio comune:

tentato omicidio (art. 56 comma 2), la pena non è inferiore a dodici anni, se la pena stabilita per il delitto è l'ergastolo, ovvero, negli altri casi, la pena è quella stabilita per il delitto, diminuita da un terzo a due terzi.

21 Cfr., tra le altre, Cass. pen., Sez. 1, sent. n. 35174 del 23/06/2009; sent. n. 37516 del 22/09/2010; sent. n. 51056 del 27/11/2013.

22 Cfr., tra le tante, Cass. pen., Sez. 1, sent. n. 35006 del 18/4/2013; sent. n. 51056 del 27/11/2013; Sez. 5, sent. n. 23618 del 11/04/2016; Sez. 1, sent. n. 11928 del 18/3/2019. 
a) l'omicidio doloso (art. 575), ${ }^{23}$ che ricorre quando è commesso con volontà omicida (nelle gradazioni del dolo diretto e del dolo eventuale); l'omicidio doloso ammette il tentativo e può essere anche plurimo; ${ }^{24}$

b) l'omicidio colposo (art. 589), che ricorre quando la morte di una persona si verifica come conseguenza non voluta di una condotta negligente, ${ }^{25} \mathrm{im}$ prudente ${ }^{26}$ o inesperta, ${ }^{27}$ oppure inosservante di leggi, regolamenti, ordini o discipline;

c) l'omicidio preterintenzionale (art. 584), che ricorre quando il soggetto agente vuole soltanto percuotere o ferire altri, ma alla sua condotta segue la morte

23 L'omicidio è doloso quando è commesso con volontà omicida, ossia con dolo intenzionale (nelle gradazioni del dolo diretto o eventuale).

Nella stragrande maggioranza dei casi, l'accertamento della volontà omicida, essendo quest'ultima il risultato di un intimo processo volitivo, si sottrae alla possibilità dell'accertamento obiettivo e diretto da parte del giudice, ma costituisce il risultato di un'indagine di fatto.

Vari possono essere gli elementi indizianti di carattere oggettivo: la natura dell'arma usata, la distanza del bersaglio dall'arma, la direzione di mira, la violenza nel colpire, la reiterazione dei colpi, la parte del corpo colpita. Se vi sono dubbi sulle modalità oggettive dell'azione, la confessione ed il movente possono acquistare rilievo come elemento secondario. In ogni caso la confessione non è di per sé prova dell'omicidio, così come l'assenza di movente non esclude senz'altro la sussistenza della volontà omicida.

24 L'omicidio volontario plurimo va tenuto distinto dal delitto di strage.

In quest'ultimo, il dolo consiste nella coscienza e volontà di porre in essere atti idonei a determinare pericolo per la vita e l'integrità fisica della collettività mediante violenza (evento di pericolo), con la possibilità che dal fatto derivi la morte di una o più persone (evento di danno), al fine (dolo specifico) di cagionare la morte di un numero indeterminato di persone, e va desunto dalla natura del mezzo usato e da tutte le modalità dell'azione (così Cass. pen., Sez. 1, sent. n. 42990 del 18/09/2008, in fattispecie nella quale la Corte ha ritenuto corretta la qualificazione di strage dell'omicidio del giudice Giovanni Falcone e della sua scorta, realizzato mediante impiego di un'enorme quantità d'esplosivo, in luogo pubblico, con effetti distruttivi di straordinaria portata).

Pertanto, al fine di stabilire se l'uccisione di più soggetti integri il delitto di strage ovvero quello d'omicidio volontario plurimo, l'indagine deve essere globale, con speciale riguardo ai mezzi usati, alle modalità esecutive del reato e alle circostanze ambientali che lo caratterizzano.

Alla stregua di detto principio (Cass. pen., Sez. 6, sent. n. 3333 del 20/11/1998), è stato ritenuto sussistente il delitto di strage (e non di omicidio volontario plurimo) nel comportamento di appartenenti a un'associazione criminosa che, dopo avere fatto irruzione in un luogo aperto al pubblico, situato nel centro cittadino e frequentato da molte persone, abbiano aperto il fuoco in maniera indiscriminata sia contro avversari non preventivamente designati sia contro persone estranee alla cosca avversaria, non rilevando che non fosse stato fatto ricorso a mezzi di natura tale (bombe o esplosivi) da cagionare la morte di un numero indeterminato di persone.

25 Si ha negligenza quando il soggetto agente compie una certa attività senza la dovuta attenzione.

26 Si ha imprudenza quando il soggetto agente tenga una condotta contraria ai generali doveri di prudenza ed accortezza.

27 Si ha imperizia quando il soggetto agente tiene un comportamento che presuppone la conoscenza di determinate regole tecniche, che tuttavia non rispetta per incapacità ovvero per inettitudine tecnica o professionale. 


\section{della persona aggredita come conseguenza non voluta (neppure nella forma eventuale ed indiretta della previsione e del rischio).}

Le suddette tre figure criminose, sotto il profilo oggettivo, sono accomunate dal fatto di consistere in un qualunque comportamento idoneo a "cagionare la morte di un uomo". 28

Tuttavia, esse si distinguono tra loro, oltre che per l'elemento soggettivo, perché possono essere caratterizzate da diverse circostanze aggravanti speciali. ${ }^{29}$

28 La morte clinica è stata a lungo identificata con l'interruzione irreversibile della respirazione e della circolazione sanguigna.

Tuttavia, i progressi della medicina, e in particolare delle tecniche di rianimazione, hanno consigliato di rivedere questa concezione ed hanno portato a spostare l'attenzione della medicina dal cuore al cervello, formulando un criterio neurologico per l'accertamento della morte. In sintesi, si sostiene che l'essere umano è morto solo quando si è avuta la cessazione irreversibile del funzionamento dell'intero encefalo: cervello, cervelletto e tronco encefalico ("morte cerebrale totale"), perché solo allora si può parlare della cessazione del funzionamento dell'organismo come un tutto.

Pertanto, l'operatore sanitario, che abbia la responsabilità professionale di accertare la morte, può basarsi sul suddetto criterio neurologico per raggiungere, caso per caso, la certezza morale, necessaria e sufficiente per attivare le procedure tecniche dirette all'espianto degli organi da trapiantare (ovviamente, previo consenso informato del donatore o dei suoi legittimi rappresentanti).

29 Ed invero:

A) Secondo gli artt. 576 e 577, le circostanze aggravanti speciali, per l'omicidio volontario, possono essere:

a) personali (la premeditazione ed i motivi abietti e futili);

b) concernenti le modalità della condotta ed i mezzi usati (l'avere adoperato sevizie o l'avere agito con crudeltà verso le persone; l'avere commesso il fatto con il mezzo di sostanze venefiche ovvero con altro mezzo insidioso);

c) concernenti la connessione tra reati (l'aver commesso il fatto per eseguire od occultare altro reato, ovvero per conseguire o assicurare a sé o ad altri il profitto o il prodotto o il prezzo ovvero l'impunità di altro reato; l'avere commesso il fatto nell'atto di compiere taluno dei delitti previsti dagli artt. 609 bis e 609 octies della legge n. 66/1996);

d) concernenti la qualità del soggetto attivo (omicidio commesso dal latitante, per sottrarsi all'arresto, alla cattura o alla carcerazione ovvero per procurarsi i mezzi di sussistenza durante la latitanza; dall'associato per delinquere, per sottarsi all'arresto, alla cattura o alla carcerazione; da persona sottoposta con provvedimento definitivo ad una misura di prevenzione);

e) concernenti i rapporti tra il colpevole e l'offeso (omicidio commesso: contro l'ascendente o il discendente; contro il coniuge, il fratello o la sorella, il padre o la madre adottivi, o il figlio adottivo, o contro un affine in linea retta).

Ai sensi dell'art. 1 della legge n. 107/1985 è omicidio aggravato anche quello commesso in danno di soggetto internazionalmente protetto.

B) D'altra parte, anche l'art. 589 c.p. prevede due aggravanti speciali per l'omicidio colposo. Invero quest'ultimo risulta aggravato:

a) qualora sia commesso con violazione delle norme per la prevenzione degli infortuni sul lavoro (comma 2): al riguardo si ricorda che il datore di lavoro è titolare di una posizione di garanzia nei confronti del lavoratore e, in quanto tale, è tenuto ad adottare tutti gli strumenti idonei a garantire la sicurezza dei propri lavoratori;

b) qualora sia commesso nell'esercizio abusivo di una professione per la quale sia richiesta una spe- 
Inoltre, l'omicidio preterintenzionale, sotto il profilo oggettivo, presenta, rispetto all'omicidio comune (doloso o colposo), quale elemento specializzante della condotta, il compimento di atti diretti a percuotere o procurare una lesione (ragion per cui, se la morte viene cagionata con atti diversi, potranno ricorrere, a seconda dei casi, i delitti previsti dagli artt. 586 e 589).

Infine, l'omicidio preterintenzionale -contrariamente all'omicidio doloso, ma al pari dell'omicidio colposo- non può sussistere nella forma tentata. ${ }^{30}$

\subsection{Due fattispecie autonome di omicidio doloso}

Il codice penale prevede altresì due fattispecie autonome, attenuate, di omicidio doloso: l'infanticidio in condizioni di abbandono materiale e morale (art. 578) e l'omicidio del consenziente (art. 579).

L'infanticidio consiste nel fatto della madre "che cagiona la morte del proprio neonato immediatamente dopo il parto (infanticidio) o del feto durante il parto (feticidio), ${ }^{31}$ quando il fatto è determinato da condizioni di abbandono materiale e morale connesse al parto". ${ }^{32}$

ciale abilitazione dello Stato oppure di un'arte sanitaria (comma 3).

Non costituisce invece una circostanza aggravante la previsione contenuta nell'ultimo comma dell'art. 589, in base al quale -qualora si verifichi la morte di più persone oppure la morte di una o più persone contestualmente alla lesione di una o più persone- si applica la disciplina del concorso formale di reati (di cui all'art. 81 c.p.), ma la pena complessiva non può comunque superare i 15 anni di reclusione (cfr. tra le tante, Cass. pen., Sez. 4, sent. n. 20340 del 7/3/2017; sent. n. 3127 del 27/01/1999).

C) Infine, secondo l'art. 585 c.p., l'omicidio preterintenzionale è aggravato:

a) se concorre una delle circostanze previste dagli artt. 576 e 577 c.p. per l'omicidio doloso;

b) se il fatto è commesso con armi o con sostanze corrosive.

30 Il tentativo non è configurabile nell'omicidio preterintenzionale per la semplice ragione che in quest'ultimo deve mancare la volontà dell'evento morte, ragion per cui l'agente, se tenta di percuotere e di ferire e cagiona la morte, risponde di omicidio; mentre, se la morte non si verifica, risponde soltanto di lesioni o percosse tentate (sempre che sia da escludere la volontà omicida).

31 Nel sistema penale italiano, il feticidio e l'infanticidio sono equiparati, ai fini dell'art. 578, ma anche ai fini degli artt. 575 e 589, in quanto nel concetto di uomo rientra sia l'infante che il feto durante il parto; ma divergono tra loro sotto il profilo temporale: il compimento della condotta feticida deve avvenire durante il parto (cioè tra il momento del distacco del feto dall'alvo materno ed il momento in cui il frutto del concepimento acquista vita autonoma), mentre il compimento della condotta infanticida deve avvenire immediatamente dopo il parto.

32 La giurisprudenza di legittimità (Cass. pen., Sez. 5, sent. n. 7756 del 26/05/1993) ha da tempo chiarito che le condizioni di abbandono materiale e morale possono ritenersi sussistenti solo quando la madre sia lasciata in balia di se stessa e venga a trovarsi al momento del parto, o subito prima o dopo di esso, in uno stato di derelizione ovvero di isolamento tale che non consente l'intervento o l'aiuto di terzi, ne' un qualsiasi soccorso fisico o morale; e che quando, invece, lo stato di abbandono materiale e morale viene volontariamente creato e mantenuto, se la morte interviene ed è collegata 


\section{L'infanticidio si distingue dall'omicidio doloso: ${ }^{33}$}

- sia per l'oggetto materiale, che non è l'uomo, ma l'uomo infante o neonato (cioè il frutto del concepimento e della gestazione, già uscito dal ventre materno, vivente e maturo per la vita extra corporea a prescindere che sia normale o deforme) ovvero l'uomo feto (cioè il frutto del concepimento e della gestazione, pervenuto alla fase del parto, vivente e maturo per la vita corporea, a prescindere che sia normale o deforme);

- sia perché è reato proprio, che può essere commesso solo dalla madre e nel quale si può al più concorrere (mentre l'omicidio è reato che può essere commesso da chiunque);

- sia per il movente, in quanto il fatto dell'infanticidio o del feticidio deve essere determinato dalle condizioni di abbandono materiale e morale, connesse al parto (altrimenti, sarebbe ravvisabile l'omicidio doloso).

\section{D'altra parte, è responsabile di omicidio del consenziente chiunque "cagiona la} morte di un uomo col consenso di lui". ${ }^{34}$ La fattispecie criminosa si distingue

causalmente a tali condizioni che hanno a loro volta determinato l'evento letale, il fatto è riconducibile all'ipotesi legislativa dell'omicidio volontario.

33 L'infanticidio si distingue altresì dall'aborto illegale sulla base del momento in cui avviene l'azione criminosa.

L'art. 19 della legge n. 194/1978 sanziona penalmente l'interruzione volontaria della gravidanza avvenuta: a) senza l'osservanza delle modalità di cui agli artt. 5 e 8 della legge in esame (fuori dalle strutture sanitarie autorizzate); b) senza l'accertamento del medico dei casi previsti dalle lettere a) e b) dell'art. 6 (interruzione dopo i 90 giorni) o senza gli accertamenti richiesti dall'art. 7 in caso di processi patologici; c) senza l'osservanza delle particolari procedure previste in caso di donne minori di anni diciotto o interdette (artt. 12 e 13).

Orbene, la condotta di colui che cagiona l'interruzione volontaria della gravidanza, prevista dall'art. 19 della legge n. 194/1978, si realizza in un momento precedente il distacco del feto dall'utero materno; mentre la condotta, prevista dall'art. 578, si realizza dal momento del distacco del feto dall'utero materno (durante il parto, se si tratta di un feto, o, immediatamente dopo il parto, se si tratta di un neonato).

Conseguentemente -è stato precisato nella giurisprudenza di legittimità (Cass. pen., Sez. 1, sent. n. 46945 del 18/10/2004) - qualora la condotta diretta a sopprimere il prodotto del concepimento sia posta in essere dopo il distacco, naturale o indotto, del feto dall'utero materno, il fatto, in assenza dell'elemento specializzante delle condizioni di abbandono materiale e morale della madre, previsto dall'art. 578, configura il delitto di omicidio volontario di cui agli artt. 575 e 577 n.1.

34 Va tuttavia precisato che non è sufficiente un qualsiasi consenso perché sia integrato l'omicidio del consenziente (in luogo del più grave omicidio comune).

Il consenso, per svolgere la sua funzione attenuatrice, deve essere: a) personale, nel senso che non è ammessa rappresentanza volontaria o legale; b) reale, nel senso che deve essere stato inequivocabilmente prestato, a prescindere dalle modalità, e non può essere presunto, anche se sottoponibile a condizione (ad es., alla condizione che venga usato mezzo indolore) ovvero a termine; c) specifico, nel senso che deve avere ad oggetto la propria uccisione; d) ed attuale, nel senso che deve essere stato prestato o comunque deve persistere al momento della condotta omicida, senza essere stato revocato. 
dall'omicidio volontario, in quanto in quest'ultimo il consenso della vittima non è elemento costitutivo del reato. ${ }^{35}$ Più precisamente: l'omicidio comune offende due beni della vittima: il bene dell'indisponibilità della vita ed il bene della libera volontà; l'omicidio del consenziente, invece, offende soltanto il bene dell'indisponibilità della vita, in quanto la volontà del fatto (anche se non necessariamente l'iniziativa) è riconducibile alla stessa vittima (nel senso che il consenso deve essere stato il fattore determinante della condotta dell'agente, che, senza di esso, non avrebbe cagionato la morte). In considerazione del fatto che il consenso della vittima rileva non soltanto ai fini della colpevolezza e della capacità a delinquere ma anche ai fini della stessa offensività del fatto, l'omicidio del consenziente è ritenuto di minore gravità, oggettiva e soggettiva, rispetto all'omicidio doloso; e, pertanto, è meno gravemente punito. ${ }^{36}$

A sua volta l'omicidio del consenziente si distingue dal delitto di istigazione o aiuto al suicidio ${ }^{37}$ che è previsto dall'art. $580^{38}$ e che si realizza: nel determi-

Inoltre, l'art. 579 comma 3, per la rilevanza del consenso, richiede che: a) il consenziente, al momento di consentire, sia persona: non minore degli anni 18; non inferma di mente; non in condizioni di deficienza psichica per altra infermità o per abuso di sostanze alcoliche e stupefacenti; b) il consenso sia determinato da motivazioni interne e, quindi, non sia estorto con violenza, minaccia o suggestione e non sia carpito con inganno.

Dunque, la richiesta di essere aiutato a morire comporta un'attenuazione della pena, soltanto nel caso in cui provenga da persona pienamente capace e libera. Al contrario, la richiesta di un minorenne, di un infermo di mente, di un soggetto in stato di deficienza psichica o ancora di chi è minacciato, ingannato o suggestionato, non ha alcun valore: chi ne ha cagionato la morte è chiamato a rispondere di omicidio volontario.

35 Rispetto a qualsiasi forma di omicidio, anche del consenziente, è sempre irrilevante il motivo, compreso quello di pietà nei confronti del soggetto passivo consenziente. Pertanto, l'eutanasia pietosa costituisce omicidio, comune o del consenziente, sempre che sia accertata l'esistenza del consenso, con i requisiti indicati nel testo.

36 Diverso è il trattamento sanzionatorio previsto: il delitto di omicidio volontario è punito con la reclusione non inferiore a 21 anni (art. 575) e, in presenza di aggravanti, anche con l'ergastolo, l'omicidio del consenziente (art. 579) è punito con la reclusione da sei a quindici anni, e l'istigazione o aiuto al suicidio (art. 580) con la reclusione da cinque a quindici anni.

37 Il suicidio, nel sistema penale italiano, viene in considerazione: a) come elemento costitutivo nei reati previsti dagli artt. 579, 580 c.p., dagli artt. 14 e 15 della legge n. 47/1948 sulla stampa e dell'art. 30 della legge n. 223/1990 sulla radiotelevisione; b) come conseguenza eventuale in altri reati (cfr. artt. 572 comma 2 e 586 c.p.); c) come eventuale mezzo per commettere il reato previsto dall'art. 640 c.p.; d) come oggetto materiale della contravvenzione di cui agli artt. 114-117 T.U. leggi p.s. Nell'ordinamento giuridico italiano, come in quello della maggior parte dei Paesi moderni, vengono punite le attività che favoriscono il suicidio (l'istigazione e l'aiuto per l'appunto), ma non è punito il suicidio. Quest'ultimo, se consumato, non viene punito per la semplice ragione che mors omnia solvit; mentre il suicidio tentato, non è punito soprattutto per motivi di opportunità pratica (la pena potrebbe agire come spinta alla programmazione del piano suicida in modo da assicurarne il successo, a non desistere o a ritentare il suicidio).

38 La Corte costituzionale con sentenza 25 settembre 2019 n. 242, emessa a seguito dell'entrata in 
nare altri al suicidio ovvero nel rafforzare l'altrui proposito al suicidio ovvero nell'agevolare in qualsiasi modo l'esecuzione dell'altrui suicidio. ${ }^{39}$ Orbene, il discrimine tra il reato di omicidio del consenziente e quello di istigazione o aiuto al suicidio va individuato -secondo la giurisprudenza di legittimità-40 nel modo in cui viene ad atteggiarsi la condotta e la volontà della vittima in rapporto alla condotta dell'agente:

- è configurabile il reato di omicidio del consenziente nel caso in cui colui che provoca la morte si sostituisca in pratica all'aspirante suicida, pur se con il consenso di questi, assumendone in proprio l'iniziativa, oltre che sul piano della causazione materiale, anche su quello della generica determinazione volitiva;

- al contrario, è configurabile il reato di istigazione o agevolazione al suicidio tutte le volte in cui la vittima abbia conservato il dominio della propria azione, nonostante la presenza di una condotta estranea di determinazione o di aiuto alla realizzazione del suo proposito, e lo abbia realizzato, anche materialmente, di mano propria.

\subsection{Due fattispecie autonome di omicidio colposo}

Due fattispecie autonome di omicidio colposo sono invece previste dalla legislazione speciale: sono l'aborto colposo e l'omicidio stradale.

L'art. 17 comma $1^{\circ}$ della legge n. 194/1978 prevede come reato la condotta di chiunque, per colpa, cagiona ad una donna l'interruzione della gravidanza (si consideri il caso del sanitario che incorra in negligenza, imprudenza o im-

vigore della legge 22 dicembre 2017 n. 219, ha dichiarato l'illegittimità costituzionale dell'art. 580 del codice penale, nella parte in cui non esclude la punibilità di chi, con le modalità previste dagli artt. 1 e 2 della suddetta legge "agevola l'esecuzione del proposito di suicidio, autonomamente e liberamente formatosi, di una persona tenuta in vita da trattamenti di sostegno vitale e affetta da una patologia irreversibile, fonte di sofferenze fisiche o psicologiche che ella reputa intollerabili, ma pienamente capace di prendere decisioni libere e consapevoli, sempre che tali condizioni e le modalità di esecuzione siano state verificate da una struttura pubblica del servizio sanitario nazionale, previo parere del comitato etico territorialmente competente".

39 L'art. 580 comma 1 parte seconda incrimina tali condotte anche se il suicidio non si verifica, sempre che dal tentativo di suicidio derivi una lesione personale grave o gravissima. L'evento, dunque, consiste alternativamente: a) nel suicidio (cioè nella autosoppressione cosciente e volontaria della propria vita mediante azione o mediante inerzia, ad es. per omessa terapia o inedia); b) nel tentativo di suicidio, con conseguente lesione personale grave o gravissima. La condotta del soggetto agente, per integrare il reato, deve costituire un contributo necessario (condicio sine qua non) o agevolatore (facilitante), a livello psichico o materiale, di uno dei suddetti eventi.

Cass. pen., Sez. 1, sent. n. 3147 del 06/02/1998. 
perizia nella prospettazione della diagnosi o nell'esecuzione di una operazione chirurgica).

La giurisprudenza di legittimità ha avuto modo di precisare che l'aborto colposo si distingue dall'omicidio colposo per il fatto che la condotta criminosa interviene prima dell'inizio del travaglio e, dunque, prima che il feto raggiunga la propria autonomia (lasciando la vita intrauterina per iniziare quella extrauterina). ${ }^{41}$

Peraltro, può essere utile ricordare che, secondo la giurisprudenza di legittimità formatasi in tema di concorso colposo del danneggiato, ${ }^{42}$ la condotta imprudente della paziente, che abbia richiesto con ritardo le cure non esclude il concorso di cause, ai sensi dell'art. 41 c.p., ove il sanitario, anziché contrastare il processo patologico in atto, differisca l'intervento terapeutico che si prospetti come necessario ed urgente alla luce di un evidente quadro clinico.

D'altra parte, l'omicidio stradale (cioè l'omicidio commesso con violazione delle norme sulla disciplina della circolazione stradale) -che costituiva in passato un omicidio colposo aggravato- a seguito dell'entrata in vigore della legge 23 marzo 2016 n. 41, ${ }^{43}$ che ha introdotto l'art. 589 bis, ${ }^{44}$ è diventato un

41 Cass. pen., Sez. 4, sent. n. 27539 del 30/01/2019 (in motivazione la Corte, richiamando le sentenze Corte cost. n. 229 del 2015 e Corte EDU, Perrillo c. Italia del 27 agosto 2015, ha precisato che deve ritenersi legittima l'inclusione dell'uccisione del feto nell'ambito dell'omicidio in considerazione dell'intervenuto ampliamento della tutela della persona e della nozione di soggetto meritevole di tutela, che dal nascituro e al concepito si è estesa fino all'embrione e che, altresì, tale inclusione non comporta una non consentita analogia in "malam partem" bensì una mera interpretazione estensiva, legittima anche in relazione alle norme penali incriminatrici).

Cfr. altresì Cass. pen., Sez. 4, sent. n. 21592 del 21/04/2016; Cass. pen., Sez. 5, sent. n. 44155 del 21/10/2008.

In particolare, tale principio è stato affermato (cfr. Cass. pen., Sez. 4, sent. n. 7967 del 29/01/2013) in fattispecie nella quale, ai fini dell'integrazione del reato di omicidio colposo, era stato ritenuto che la morte era sopraggiunta a travaglio iniziato quando il feto, benché ancora nell'utero, aveva raggiunto una propria autonomia con la rottura del sacco contenente il liquido amniotico.

42 Cass. Pen., Sez. 5, sent. n. 10482 del 14/07/2000 (in fattispecie nella quale la Corte ha ritenuto che correttamente i giudici di merito avessero affermato l'efficacia causale della condotta del medico il quale aveva avviato a diverso nosocomio una paziente che, sia pur con ritardo, aveva richiesto le cure per il distacco della placenta, così omettendo un immediato intervento che avrebbe potuto salvare la vita del feto).

43 Il legislatore italiano ha ritenuto di prevedere per l'omicidio stradale una disciplina differenziata rispetto alle altre tipologie di omicidio, in considerazione del gran numero di incidenti mortali che si verificano in Italia, causati soprattutto dalla disattenzione e dalla guida sotto effetto di sostanze alcoliche e droganti.

44 Il legislatore italiano, introducendo l'art. 589 bis c.p., da un lato, ha voluto sottrarre la precedente disposizione normativa (ovvero la previsione dell'omicidio colposo stradale come mera aggravante dell'omicidio colposo ex art. 589) al giudizio di bilanciamento di cui all'art. 69 c.p.; e, dall'altro, ha inteso ovviare all'orientamento giurisprudenziale secondo il quale la condotta di chi guidi in stato 
reato autonomo rispetto all'omicidio colposo, con pene più severe e diverse circostanze aggravanti.

Invero, l'art. 589-bis punisce oggi chi cagiona "per colpa" la morte di una persona, a seguito di violazione delle norme sulla disciplina della circolazione stradale. Trattasi, pertanto, di una fattispecie qualificata di omicidio colposo, che prevede una pena edittale più alta (da due a sette anni) rispetto a quella di cui al primo comma dell'art. 589 (da sei mesi a cinque anni). ${ }^{45}$

Sono previste, inoltre, delle circostanze aggravanti speciali (quali, ad esempio, la commissione del fatto sotto l'effetto di sostanze alcooliche o stupefacenti o con violazioni particolarmente gravi del codice della strada: elevata velocità all'interno delle aree urbane, guida contromano, attraversamento con il semaforo rosso ecc.).

Ulteriore aumento di pena è previsto dall'art. 589-ter, nel caso in cui il responsabile dell'incidente mortale si dia alla fuga.

Inoltre, al fine di responsabilizzare maggiormente chi si mette alla guida, l'art. 590-quater statuisce che, ove ricorrano le citate circostanze aggravanti, eventuali circostanze attenuanti non possono mai essere ritenute, nel giudizio di bilanciamento, equivalenti o prevalenti.

\title{
4.4. Omicidio preterintenzionale e morte come conseguenza di altro delitto
}

L'omicidio preterintenzionale costituisce fattispecie speciale rispetto al reato

\begin{abstract}
di ebbrezza non può essere sussunta in una ipotesi di omicidio volontario nella forma del dolo eventuale. Infatti, aderendo alle recenti teorie volitive, si era negato che l'agente potesse rispondere a titolo di dolo, dato che, pur essendo la sua condotta riprovevole, non può certo dirsi che egli avesse in qualche modo accettato l'evento morte. Tutt'al più, in tali casi, poteva configurarsi l'accettazione del rischio dell'evento, in seguito alla mera previsione di esso, ma questa ipotesi rientra nel concetto di colpa aggravata dalla previsione dell'evento (art. 61, n. 3 c.p.).

La giurisprudenza di legittimità è da anni consolidata nel ritenere che linea di demarcazione tra dolo eventuale e colpa con previsione va individuata nel diverso atteggiamento psicologico dell'agente che, nel primo caso, accetta il rischio che si realizzi un evento diverso non direttamente voluto mentre nella seconda ipotesi nonostante l'identità di prospettazione, respinge il rischio, confidando nella propria capacità di controllare l'azione (Cass. pen., Sez. 4, sent. n. 11024 del 10/10/1996). Comune è, pertanto, la previsione dell'evento diverso da quello voluto mentre ciò che diverge è l'accettazione o l'esclusione del rischio relativo. Trattasi di atteggiamenti psicologici che vanno ricostruiti affidandosi agli elementi sintomatici evidenziati dal comportamento del soggetto.

45 Peraltro, il tenore letterale dell'art. 589-bis non consente di applicare tale norma in caso di omicidio stradale avvenuto a seguito di azione dolosa, con la conseguenza che -ove l'elemento psicologico dell'autore di un incidente stradale con esito mortale sia qualificabile come dolo, sia pure eventuale- la fattispecie realizzata non potrà essere ricondotta a quella di omicidio stradale, bensì alla diversa ipotesi dell'omicidio volontario, punito ai sensi degli artt. 575 ss.
\end{abstract}


previsto dall'art. 586 (morte come conseguenza di altro delitto), che ricorre quando da un fatto, preveduto come delitto doloso, deriva, quale conseguenza non voluta dal soggetto agente, la morte o la lesione di una persona: ${ }^{46}$ invero, nell'art. 586, la morte deve essere conseguenza non voluta di un qualsiasi delitto doloso (tentato o consumato); mentre, nell'art. 584, la morte deve essere conseguenza non voluta di un delitto di percosse o di lesione (tentato o consumato).

In altri termini, nel delitto previsto dall'art. 586, l'attività del colpevole è diretta a realizzare un delitto doloso diverso dalle percosse o dalle lesioni personali, mentre, nell'omicidio preterintenzionale, il soggetto agente pone in essere una condotta finalizzata a realizzare un evento che, ove non si verificasse la morte, costituirebbe un reato di percosse o lesioni. ${ }^{47}$ Dunque, nell'omicidio preterintenzionale, l'agente vuole ledere un bene giuridico (quello della incolumità personale) che appartiene allo stesso genere del bene protetto dall'omicidio (quello della vita), dal quale si distingue per la progressione dell'offesa; mentre, nel delitto previsto dall'art. 586, l'agente finisce con l'offendere un bene giuridico completamente diverso e, quindi, pone in essere un delitto di "specie" diversa.

\subsection{Considerazioni di sintesi}

Tutti i suddetti delitti sono a condotta libera: ${ }^{48}$ ai fini della loro sussistenza, la legge non richiede il compimento di specifiche condotte, ma solo che esista un nesso di causalità tra la condotta e la morte, oltre alla volontà di procurarla

46 Dunque, il reato previsto dall'art. 586 c.p. integra una ipotesi di concorso formale di un delitto doloso e di un delitto di omicidio colposo (o di lesioni colpose), che va considerato nei requisiti costitutivi e negli effetti come una qualsiasi ipotesi di concorso formale. Per questa ragione, si tratta di una disposizione sostanzialmente inutile, che, per esigenze di semplificazione, dovrebbe essere soppressa in una prospettiva de iure condendo.

47 Cass. pen., Sez. 5, sent. n. 23606 del 04/04/2018.

48 In conformità a detto principio, è stato ravvisato l'omicidio preterintenzionale in relazione ad un decesso causato da un'azione violenta consistita nell'immobilizzare ed imbavagliare la vittima e dalla quale era derivato un fenomeno di asfissia da soffocamento (Cass. pen., Sez. 5, sent. n. 21002 del 20/04/2015); mentre, in tema di morte o lesioni come conseguenza di altro delitto (Cass. pen., Sez. 6, sent. n. 49573 del 19/09/2018), è stato affermato che "la morte dell'assuntore di sostanza stupefacente è imputabile alla responsabilità del cedente sempre che, oltre al nesso di causalità materiale, sussista la colpa in concreto per violazione di una regola precauzionale (diversa dalla norma che incrimina la condotta di cessione) e con prevedibilità ed evitabilità dell'evento, da valutarsi alla stregua dell'agente modello razionale, tenuto conto delle circostanze del caso concreto conosciute o conoscibili dall'agente reale".

Anche l'omicidio preterintenzionale è un reato a forma libera, ma postula comunque il verificarsi di una condotta di percosse o di lesioni. 
(si può uccidere cioè un'altra persona con un veleno, oppure con un'arma, o spingendola giù da un precipizio).

Sono punite anche le omissioni: quando il soggetto provoca la morte di un uomo non effettuando determinate azioni che ne avrebbero salvato la vita. Tuttavia, per non allargare enormemente il numero dei potenziali responsabili di una morte, è penalmente responsabile soltanto chi era giuridicamente obbligato ad un certo comportamento attivo (in forza di una legge o di un altro atto che crea un obbligo giuridico) e non l'ha messo in atto (art. 40 c.p. "Non impedire un evento, che si ha l'obbligo giuridico di impedire, equivale a cagionarlo"): ad esempio, i genitori, che hanno il dovere di mantenere i figli (articolo 147 c.c., articolo 30 Cost.), sono responsabili se un figlio, non nutrito o non curato dalle malattie da cui è affetto, muore; così come il medico, obbligato in forza del rapporto contrattuale con il paziente o comunque del suo ruolo (ad esempio all'interno di un ospedale), se omette volontariamente terapie o forme di sostegno vitale, è responsabile per omicidio volontario del paziente che muore; così come, ancora, l'infermiere, limitatamente ai suoi obblighi, o chi ha la cura e la custodia di una persona incapace di provvedere alle proprie necessità.

Le cause di non punibilità (cd. scriminanti) delle condotte di omicidio volontario sono quelle generali: in particolare la legittima difesa ( $\mathrm{ad}$ es., non è punibile chi uccide un rapinatore che lo sta assalendo) e lo stato di necessità (ad es., non è punibile chi, trovandosi con altra persona in un luogo isolato e con pochissimo cibo, lascia morire di fame quest'ultima per potersi nutrire soltanto lui e potersi, quindi, così salvare). Non sono, invece, applicabili altre cause di giustificazione della condotta. In particolare non è applicabile la causa di giustificazione del consenso dell'avente diritto (art. 50 c.p.): il divieto di omicidio del consenziente dimostra che la vita umana è un diritto indisponibile e, quindi, la richiesta di essere ucciso non rende lecito l'atto. D'altra parte, nell'ordinamento giuridico italiano, non viene riconosciuto a nessuno il diritto ad uccidere un uomo: ${ }^{49}$ ad esempio, la famiglia di una persona uccisa non ha il diritto di vendicarsi, uccidendo a sua volta il colpevole, né il coniuge tradito ha il diritto di uccidere l'altro coniuge e il suo amante. Tanto meno è configurabile un obbligo giuridico di uccidere in tempo di pace; questo obbligo grava soltanto sui soldati in guerra, ma l'ordinamento italiano ormai da vari anni riconosce

49 Salvo gli agenti di polizia che agiscano per impedire la consumazione di gravi reati: a scriminare il loro operato valgono le cause di giustificazione della legittima difesa -che vale anche per difendere diritti altrui- o dell'uso legittimo delle armi (cf. art. 53). 
l'obiezione di coscienza al servizio militare obbligatorio e, quindi, permette di esserne esentati. ${ }^{50}$

In definitiva, l' ordinamento giuridico italiano, civile e penale: vieta di cagionare la morte di un uomo, anche se con il suo consenso; attribuisce rilevanza penale alla condotta di chi determina altri al suicidio o rafforza l'altrui proposito di suicidio, oppure ne agevola in qualche modo l'esecuzione; non permette che i neonati -a prescindere che siano sani o malati- vengano lasciati morire; non gradua il diritto a vivere a seconda delle condizioni di salute delle persone; aborra ogni forma di uccisione motivata su base genetica o razziale o etnica; riconosce uguale natura umana ad ogni persona.

\section{La dimensione relazionale dei diritti individuali della persona}

La storia del costituzionalismo moderno insegna che il diritto alla vita ed alla incolumità personale sono stati riconosciuti ed affermati fin dalle prime carte dei diritti del secolo XVIII per proteggere la persona umana dall'arbitrio e dagli abusi dei potenti. D'altronde, lo jus della tradizione giuridica dell'Europa occidentale è essenzialmente regolamentazione di un rapporto: è noto il suum cuique tribuere che il giurista romano Eneo Domizio Ulpiano ha lasciato scritto nelle sue Regole ormai molti secoli fa.

Con ciò intendo dire che ogni diritto individuale (e, tra tutti, il diritto alla vita) non riguarda soltanto la persona umana, che ne è il titolare, ma riguarda necessariamente il complessivo intreccio relazionale, nel quale ciascuna persona umana è inserita, con la conseguenza che in ogni singolo rapporto deve essere tenuto in considerazione anche il soggetto passivo, a sua volta portatore della dignità propria di tutti gli esseri umani.

La dimensione relazionale dei diritti della persona umana è generalmente sottesa nell'ordinamento giuridico italiano (dalla disciplina dei rapporti patrimoniali a quella dei rapporti successori; dalla disciplina dell'impresa a quella del lavoro, pubblico e privato, ecc.).

50 Per poliziotti e carabinieri, vige la scriminante dell'ordine dell'Autorità (art. 51): "Se un fatto costituente reato è commesso per ordine dell'Autorità, del reato risponde sempre il pubblico ufficiale che ha dato l'ordine. Risponde del reato altresì chi ha eseguito l'ordine, salvo che, per errore di fatto, abbia ritenuto di obbedire ad un ordine legittimo. Non è punibile chi esegue l'ordine illegittimo, quando la legge non gli consente alcun sindacato sulla legittimità dell'ordine". La legge n. 121 del $1^{\circ}$ aprile 1981, in tema di "Nuovo ordinamento dell'Amministrazione della pubblica sicurezza", prevede che il poliziotto "al quale venga impartito un ordine la cui esecuzione costituisce manifestamente reato, non lo esegua e informi immediatamente i superiori”. 
Eppure non sempre ricorre nel delicato settore del diritto delle persone.

Tale dimensione è sì presente nel settore del diritto minorile: i minori d'età, per la nostra come per tutte le legislazioni contemporanee, sono privi del potere dispositivo sui propri beni e della pienezza di riconoscimento dei loro atti di libertà (e non solo in campo patrimoniale). Come è stato acutamente osservato ${ }^{51}$ l'assetto giuridico della minore età è la prova più convincente della preferibilità della dottrina (oggettiva) dell'interesse rispetto alla dottrina (soggettiva) della volontà, nel riconoscimento dei diritti individuali. Il criterio del "best interests" -assurto a rango di norma internazionale con la Convenzione ONU sui Diritti del Fanciullo del 1989, e tradotto in molte lingue come "superiore interesse del minore" ${ }^{52}$ allude proprio al fatto (curioso e provocatorio per le dottrine soggettiviste e libertarie) che l'interesse del minore non coincide di per sé: né con quanto vogliono i genitori del minore (spesso, peraltro, in disaccordo tra loro) o comunque i soggetti che sul minore esercitano la potestà genitoriale; né con quanto il minore stesso ritiene suo superiore interesse; ma va ricercato nel concreto intreccio relazionale nel quale il minore vive: incombenza questa rimessa alla competenza professionale ed alla capacità di introspezione psicologica del giudice minorile (che deve poi dar conto in motivazione delle argomentazioni, logiche e giuridiche, poste a base della decisione). ${ }^{53}$

51 Nel celebre saggio di MacCormick (1976, pp. 305-317).

52 Il principio della tutela del "superiore interesse del fanciullo" trova solenne proclamazione nell'art. 3 dalla Convenzione sui Diritti del fanciullo, approvata dall'Assemblea generale delle Nazioni Unite il 20 novembre 1989 a New York (ratificata dall'Italia con Legge 27.05.1991, n. 176), in base al quale: "In tutte le decisioni relative ai fanciulli, di competenza delle istituzioni pubbliche o private di assistenza sociale, dei tribunali, delle autorità amministrative o degli organi legislativi, l'interesse superiore del fanciullo deve essere una considerazione preminente".

Tale disposizione è stata ribadita anche da altre normative (e, in particolare, dall'art. 24 della Carta dei diritti fondamentali dell'Unione europea; e negli artt. 1 comma 2, 6 lett. a), e 10 della Convenzione EDU) e dalla giurisprudenza a livello internazionale, fino a diventare un principio generale ormai consolidato all'interno dell'ordinamento giuridico di numerosi Stati.

All'interno dell'ordinamento giuridico del nostro Paese, la Consulta riconosce pacificamente al principio del "superiore interesse del bambino" rilievo costituzionale (cfr., tra le tante, le sentenze nn. 76/2017, 308/2008, 61/2006, 425/2005 e 341/1990). Tale principio viene inquadrato alla stregua di vera e propria clausola generale che, alla luce degli artt. 2 e 31 Cost., costituisce parametro di valutazione della costituzionalità delle leggi.

Il principio del "superiore interesse del bambino" viene tra l'altro ribadito anche a livello di legislazione ordinaria: in special modo - ma non solo - nel codice civile (artt. 158, comma 2, 250, commi 4 e 5, 251, comma 1, 252, commi 2 e 5, 317 bis, comma 2, 336 bis, comma 1, 337 ter, comma 2, 337 quater, comma 2, 337 sexies, comma 1, 337 octies, comma 1) e nella disciplina dell'adozione (artt. 4, commi 5 e 5 ter, 11, comma 1, 14, comma 2, 25, commi 3, 4 e 5, 32, comma 1, 33, comma 4, 35, commi 3 e 4, 43, comma 3, 46, comma 2, 57, comma 1, n. 2, l. 4 maggio 1983, n. 184).

53 Uno dei punti più delicati della trattazione del concetto di "best interest of the child" riguarda la tradu- 
Ma la dimensione relazionale dei diritti individuali non sembra affatto presente nel particolare settore dell'inizio vita, dove parte della cultura contemporanea è riuscita ad oscurare la percezione, personale e collettiva, del valore della vita umana: in questo particolare settore, la legislazione italiana, come molte altre legislazioni contemporanee, consente alla donna di richiedere l'interruzione volontaria di gravidanza (IVG) entro i primi 90 giorni di gestazione per motivi di salute, economici, sociali o familiari, senza farsi carico di considerare che al c.d. diritto della donna ad autodeterminarsi ${ }^{54}$ (e, in particolare, a prendere decisioni riguardo al proprio corpo) fa fronte il diritto alla vita dell'embrione.

La biologia dimostra che la vita di un nuovo essere umano inizia nel momento della fecondazione, ${ }^{55}$ ossia nella fusione tra lo spermatozoo maschile e l'ovulo femminile. L'unione di 23 cromosomi del gamete maschile con 23

zione della locuzione in lingua italiana. La traduzione dell'art. 3 della Convenzione ONU relativo alla legge di ratifica parla di "protezione del miglior interesse del minore", mentre in altri casi si preferisce utilizzare il termine "superiore", ovvero "preminente", piuttosto che il termine "prevalente", sempre in riferimento alla tutela dell'interesse del fanciullo. Il punto fondamentale concerne la valenza che si desidera conferire al concetto nel bilanciamento con gli altri interessi in gioco, nel senso che attraverso questo termine il giudice decide che l'interesse del fanciullo debba prevalere sempre oppure possa essere commisurato con altri valori di pari dignità costituzionale, almeno in via astratta.

La Suprema Corte italiana ha da anni chiarito che "le indagini e le valutazioni del giudice del merito non debbono svolgersi sul piano astratto e generale, ma, in ossequio al principio di rilevanza costituzionale della tutela del prevalente interesse del minore, debbono avere ad oggetto la fattispecie concreta" (Cass. Civ., Sez. I, 16.02.2002, n. 2303); mentre, più di recente, ha richiamato il suo "costante orientamento... in merito alla necessità di un accertamento in concreto dell'interesse del minore nelle vicende che lo riguardano, con particolare riferimento agli effetti del provvedimento richiesto in relazione all'esigenza di uno sviluppo armonico dal punto di vista psicologico, affettivo, educativo e sociale" (Cass. civ., sez. I, 22 dicembre 2016, n. 26767).

54 A mio avviso, il diritto all'autodeterminazione della donna è evocato a sproposito. In materia, l'autodeterminazione della donna e dell'uomo consiste nel prendere liberamente la scelta di concepire oppure di non concepire, ma non nella possibilità di sopprimere il concepito, perché in questo caso c'è di mezzo la vita di un terzo essere umano. Purtroppo, dietro il sostegno in favore dell'autodeterminazione della donna, si nasconde spesso il tornaconto di coloro che nei confronti della donna non vogliono assumersi le proprie responsabilità.

D'altronde, come può esistere il diritto di non riconoscere che qualsiasi altro essere umano, altro rispetto a noi, in quanto essere umano, è uguale a noi in tutto ciò che riguarda il diritto alla vita? Il fatto è che non può esistere un diritto al non riconoscimento dell'altro come uguale a noi, perché tale riconoscimento è la regola fondamentale del diritto e della giustizia. Al contrario, riconoscere che essere umano, per il semplice fatto di essere tale, è uguale a noi è principio sotteso al divieto di ogni forma di discriminazione: razziale, religiosa, economica, eugenetica, ecc.

55 Al riguardo, è illuminante la relazione resa nel 1989 dal Prof. Jérome Lejeune, nella sua qualità di genetista, ad un processo a Maryville (Tennessee, USA), e successivamente raccolta nel volume L'embrione segno di contraddizione. Dai verbali del processo di Maryville, edito nel 1992 dalle Edizioni Orizzonte Medico. E già il mondo pagano vedeva nel nascituro un soggetto giuridico (è di Tertulliano il felice aforisma: Homo est et qui est futurus: etiam fructus omnis iam in semine est: Apologeticum IX, 8). 
cromosomi del gamete femminile produce una nuova cellula di 46 cromosomi. Questa nuova cellula, detta zigote, contiene un nuovo codice genetico, che produce un individuo umano differente dal padre, dalla madre e da ogni altra persona del mondo. Sul piano biologico, lo zigote è, sia pure in miniatura, un essere umano nuovo, unico e completo, ${ }^{56}$ che inizia a vivere, anche se con potenzialità ancora inattuate ed anche se incapace di vita indipendente. ${ }^{57}$

Confesso che più volte mi sono chiesto come mai nella nostra società è diffusa la consapevolezza che non è giusto operare discriminazioni sulla base del colore della pelle ovvero sulla base di convinzioni politiche o religiose, mentre è assai debole la consapevolezza che non è giusto discriminare il diritto fondamentale alla vita di alcuni esseri umani già concepiti per il semplice fatto che non sono nati, che non sono desiderati o che non possiedono la capacità di rivendicare i loro diritti.

Oltretutto, il principio dell'inviolabilità assoluta della vita umana innocente risponde ad uno dei valori sostanziali (la vita) e ad uno dei principi fondamentali (il principio di uguaglianza) che stanno alla base dello Stato democratico e costituzionale moderno. ${ }^{58}$ Ed il diritto alla vita è uno dei pochi diritti della persona umana che, secondo il sistema realizzato dalla Convenzione europea dei diritti dell'uomo, dovrebbe non poter essere derogato da alcun altro diritto (e, pertanto, dovrebbe sfuggire a qualsiasi operazione di bilanciamento). ${ }^{59}$

56 Lo zigote è un essere umano unico, in quanto non è mai esistito in passato e non esisterà mai in futuro un essere umano identico a lui. Ed è un essere umano completo, in quanto il codice genetico (genotipo) dello zigote contiene l'informazione su tutte le sue caratteristiche (statura, colore degli occhi, dei capelli e della pelle, ecc.).

57 Si consideri che non solo i nascituri, ma anche i neonati sono incapaci di vita indipendente: senza la costante cura da parte della madre o di altre persone che lo assistono, il neonato non sopravvive e muore ben presto.

58 Norberto Bobbio rispondeva, a chi si richiamava al patto sociale, "che il primo grande scrittore politico che formulò la tesi del contratto sociale, Tommaso Hobbes, riteneva che l'unico diritto cui i contraenti entrando in società non avevano rinunciato era il diritto alla vita" (intervista rilasciata da N. Bobbio a "La Stampa" del 15-V-1981 e richiamata da Palini, 1992, p. 74)

59 La circostanza si spiega per la seguente semplice ragione: il diritto alla vita è un diritto primario, anzi, è il diritto primario per eccellenza, senza il quale non è possibile esercitare nessun altro diritto; esso, dunque, va difeso più e prima di ogni altro diritto.

Una madre, che vuole abortire, pretende di esercitare un proprio diritto secondario e derivato -quale per l'appunto quello di "gestire il proprio corpo" o di liberarsi da un "problema"- sacrificando il diritto, primario e originario, che il figlio ha di vivere. Non si tratta di colpevolizzare la donna partoriente -che anzi il più delle volte va difesa, perché subisce l'aborto sotto le pressioni del partner, della famiglia o dell'ambiente sociale in cui vive- ma non si può pretendere di ottenere un proprio (discutibile) vantaggio, facendolo pagare ad altri, peraltro al carissimo prezzo della vita. Non si tratta neppure di costringere la madre ad accettare un figlio non desiderato, ma non si può pretendere che il proprio rifiuto si traduca in una uccisione legalizzata. 
D'altronde, la posta in gioco è così alta che, quand'anche fosse soltanto dubbio che l'embrione umano sia una presenza personale, non dovrebbe comunque potersi consentire un intervento volto a sopprimerlo (giacché in dubio, pro vita).

Il fatto è che sulla legalizzazione dell'interruzione volontaria della gravidanza si è formato oggi un consenso sociale così largo che neppure le giurisdizioni, nazionali e sovranazionali, che dovrebbero essere deputate alla tutela dei diritti della persona umana (e, tra questi, ribadisco, i diritti dell'essere umano nascituro) riescono a contrastare la tirannide della maggioranza (di cui non di rado le stesse giurisdizioni superiori sono espressione). ${ }^{60}$

Considerazioni analoghe valgono per il trattamento che molte legislazioni nazionali riservano al fine vita. ${ }^{61}$

Si dimentica o si ignora che, come insegnava Norberto Bobbio (1983), 62 "la regola della maggioranza non è applicabile a tutte le situazioni” (p. 18).

I legislatori nazionali, piuttosto che intervenire sull'interruzione della gravidanza (per legalizzarla o per criminalizzarla), dovrebbero rafforzare le diverse forme di assistenza di minori abbandonati, l'adozione e l'affido. Peraltro, così operando, verrebbero incontro al desiderio di molte coppie che fanno ricorso (talvolta ripetutamente e inutilmente) alla fecondazione artificiale, sostenendo anche spese impegnative, pur di riuscire ad avere un figlio. In ogni caso, la soppressione di un essere umano non può essere un modo per risolvere problemi, che devono trovare altrove la loro soluzione.

60 Il ragionamento che suole farsi è per lo più il seguente: se tu non sei d'accordo, non agire così, ma non imporre agli altri la tua opinione.

Senonché, occorre convincersi che quanto avviene nell'intimità dell'utero materno non è una faccenda privata della donna, ma è la formazione e lo sviluppo di un essere umano, che ha pieno diritto alla protezione legale.

Può aggiungersi che una società, che non conosca limiti, finisce con il tradire l'uomo, perché, se non ci sono limiti, deve intendersi permessa ogni cosa (e, seguendo questo tipo di argomentare, si potrebbe giungere a giustificare anche le inique condizioni lavorative o sociali, spesso tanto diffuse anche ai giorni nostri).

61 Nella società occidentale contemporanea si sta diffondendo sempre di più la convinzione che: le persone con handicap, soprattutto se mentale, sono inutili, se non dannose alla società; il cosiddetto stato vegetativo non costituisce una condizione degna di essere chiamata vita ed, anzi, è doveroso, per il bene della società, impedire la diffusione delle patologie genetiche mediante l'eliminazione dei portatori; uccidere un neonato sfortunato o che rischia di vivere con un handicap sia un bene per lo stesso neonato; soggetti in totale stato di demenza -privi di autocoscienza- non possono essere chiamati e comunque non hanno il diritto di essere definiti persone; la condizione di sofferenza derivante da una malattia inguaribile non può essere sopportata; gli anziani (soprattutto se poveri) sono un costo, se non uno spreco per la società (in termini di occupazioni di posti-letto, costi delle terapie ecc.), ecc. Il punto problematico è che, essendo venuto meno nel contesto sociale un quadro antropologico di riferimento, non può più ritenersi pacifico che la dignità è una caratteristica intrinseca di ogni vita umana, essendo da molti sostenuto che essa sia una qualità che può anche non ricorrere in alcune forme di esistenza umana.

62 Secondo Bobbio (1983) -comunemente considerato uno dei massimi teorici del diritto e nel contempo uno dei massimi filosofi della politica nella seconda metà del Novecento- non possono essere sottoposti a un voto della maggioranza "il diritto alla vita, alla libertà, alle varie forme di libertà personale, civile e sociale”, in quanto detti diritti costituiscono le condizioni necessarie per l'applicabilità stessa del principio maggioritario. 
Ecco dunque che, per non trasformare in diritto ogni interesse individuale, occorre mantenere una visione globale del reale; e non dimenticare che il diritto regola rapporti intersoggettivi (jus hominum causa constitutum est). ${ }^{63}$

Solo recuperando l'idea del diritto come rapporto, propria della tradizione giuridica dell'Europa occidentale, si può comprendere il carattere ideologico di una certa "logica dei diritti individuali" e convincersi che, rispetto ad ogni nuovo preteso diritto individuale, occorre comprendere se l'interesse, che esso sottende, assume una rilevanza che giustifichi la sua attrazione nella sfera della giuridicità, cioè nella dimensione relativa alla disciplina dei rapporti all'interno di un gruppo socialmente organizzato. ${ }^{64}$ In altri termini, occorre cercare di com-

In una prospettiva di etica politica, occorre domandarsi sulla base di quale fondamento razionale lo Stato moderno - che è sorto proprio per garantire beni, come la vita e la salute - possa porre obblighi, limitativi di tali beni, a carico delle strutture sanitarie pubbliche.

63 Va altresì considerato che trasformare in diritto pretese soggettive individuali implica necessariamente prevedere obblighi a carico di altri. Ciò è imposto dalla realtà delle cose: ogni diritto ha un costo per la società, in termini di risorse necessarie per la sua attuazione e per la sua tutela nonché in termini di sacrificio di altri interessi con esso confliggenti. Ragion per cui, se io, ad es., affermo il mio diritto a guidare la moto senza casco, come diritto a muovermi come voglio, anche a costo, nel caso di incidente stradale, di subire gravi lesioni o di mettere a repentaglio la mia stessa vita, affermo la preminenza di tale mio desiderio sull'interesse dello Stato a prevenire le ingenti spese sanitarie connesse agli incidenti stradali e sull'interesse che altri hanno all'adempimento dei miei doveri, in quanto membro di una comunità di persone legate da reciproci rapporti di solidarietà. E' questo un aspetto che la completezza di un'argomentazione giuridica non può eludere, mentre la c.d. "argomentazione per diritti" tende a non far emergere: la logica individualista dei "diritti-desiderio" occulta il fatto che trasformare in diritto una pretesa soggettiva, in ipotesi anche apprezzabile, implica sempre affermare obblighi a carico di altri.

64 Sulla necessità di "prestare attenzione per non cadere in alcuni equivoci che possono nascere da un fraintendimento del concetto di diritti umani e da un loro paradossale abuso", Papa Francesco, nel discorso al Parlamento europeo, pronunciato a Strasburgo il 25 novembre 2014 (disponibile sul sito istituzionale www.vatican.va), si è così espresso:

"Vi è oggi la tendenza verso una rivendicazione sempre più ampia di diritti individuali -sono tentato di dire individualistici- che cela una concezione di persona umana staccata da ogni contesto sociale e antropologico, quasi come una 'monade' (uovós), sempre più insensibile alle altre 'monadi' intorno a sé. Al concetto di diritto non sembra più associato quello altrettanto essenziale e complementare di dovere, così che si finisce per affermare i diritti del singolo senza tenere conto che ogni essere umano è legato a un contesto sociale, in cui i suoi diritti e doveri sono connessi a quelli degli altri e al bene comune della società stessa.

Ritengo perciò che sia quanto mai vitale approfondire oggi una cultura dei diritti umani che possa sapientemente legare la dimensione individuale, o, meglio, personale, a quella del bene comune, a quel 'noi-tutti' formato da individui, famiglie e gruppi intermedi che si uniscono in comunità sociale. Infatti, se il diritto di ciascuno non è armonicamente ordinato al bene più grande, finisce per concepirsi senza limitazioni e dunque per diventare sorgente di conflitti e di violenze. Parlare della dignità trascendente dell'uomo, significa dunque fare appello alla sua natura, alla sua innata capacità di distinguere il bene dal male, a quella 'bussola' inscritta nei nostri cuori e che Dio ha impresso nell'universo creato; soprattutto significa guardare all'uomo non come a un assoluto, ma come a un essere relazionale". 
prendere se il preteso nuovo diritto individuale si collochi, secondo ragione, madre di ogni diritto, entro gli argini di una "esperienza elementare universale", ${ }^{65}$ riconoscibile per ogni persona umana.

\section{Bibliografía}

Bobbio, N. (1983). La regola della maggioranza e i suoi limiti. En Bobbio N., Bodei, R., Marramao, G., Rella, F., Rovatti, P. A., Schiera, P. y Stame, F. (eds.), Soggetti e potere. Un dibattito su società civile e crisi della politica. Bibliopolis.

Cartabia, M. (2008). Gli strumenti di tutela dei diritti fondamentali. Informe presentado en la conferencia Istituzioni Pubbliche e Garanzie del Cittadino. Milán, Italia, 3 y 4 de julio.

MacCormick, N. (1976). Children's Rights: A Test-Case for Theories of Right. Archiv für Rechtsund Sozialphilosophie / Archives for Philosophy of Law and Social Philosophy, 62(3), 305-317.

Palini, A. (1992). Aborto. Dibattito sempre aperto da Ippocrate ai nostri giorni. Città Nuova Editrice.

65 La felice espressione è di Marta Cartabia (2008) (già Presidente della Corte costituzionale italiana). 